\title{
Estimation Of The Quality Of Life Benefits Associated With Treatment For Spinal Muscular Atrophy
}

This article was published in the following Dove Press journal:

ClinicoEconomics and Outcomes Research

\author{
Andrew J Lloyd (iD) \\ Robin Thompson ${ }^{2}$ \\ Katy Gallop' \\ Megan Teynor ${ }^{3}$ \\ 'Acaster Lloyd Consulting Ltd., London, \\ UK; ${ }^{2}$ Value and Access, Biogen, Baar, \\ Switzerland; ${ }^{3}$ Value and Access, Biogen, \\ Cambridge, MA, USA
}

Background: Spinal muscular atrophy (SMA) is a rare, genetic, progressive neuromuscular disorder characterized by severe muscle atrophy and weakness and is a leading genetic cause of death in infants and children. Nusinersen was the first treatment targeting the underlying cause of disease approved by the FDA, EMA and other countries for patients with SMA. There are currently very limited data available on the health-related quality of life (HRQoL) burden of SMA suitable for use in a cost-effectiveness analysis.

Objective: This study was designed to estimate quality of life weights or utilities for different SMA states.

Methods: SMA case studies were developed describing Type I (infantile onset) and Type II (later-onset) patients and different outcomes from treatment. These were developed so that quality of life weights or utilities (where the value of health ranges from 1 - full health to 0 dead) could be estimated for cost-effectiveness analysis. Clinical experts $(n=5)$ rated each of the case studies using standardized HRQoL instruments - the EQ-5D-Y and PedsQL-NMM (baseline states only).

Results: The SMA Type I utilities ranged from -0.33 (requires ventilation) to 0.71 (Type I patient reclassified as Type III following treatment), with quite substantial differences between some states. Most Type I states had a utility score below zero indicating the severity of the states. The SMA Type II utilities ranged from -0.13 (worsened) to 0.72 (stands/walks unaided). In general, the results showed HRQoL improved in line with better health states.

Conclusion: The utility scores obtained in this study highlight the very substantial burden experienced by SMA patients. Despite the limitations in the methods used, this study produced data with face validity and is a useful starting point for understanding the burden of SMA Types I and II in cost-effectiveness analysis.

Keywords: spinal muscular atrophy, health-related quality of life, utility, EQ-5D, costeffectiveness analysis

\section{Introduction}

Spinal muscular atrophy (SMA) is an autosomal recessive neuromuscular disease with an estimated incidence rate $8.5-10.3$ per 100,000 live births. ${ }^{1}$ Life expectancy is strongly correlated with age of onset ${ }^{2}$ with the most severe cases often not living beyond two years of age without respiratory support and nutritional interventions. SMA is characterized by degeneration of alpha motor neurons in the spinal cord, resulting in progressive proximal muscle weakness and atrophy. ${ }^{3}$

SMA is classified into four phenotypes based on age at onset and motor function achieved. SMA Type I accounts for more than half of all new SMA cases; ${ }^{4}$ however, the prevalence has been shown to be $0.04-0.28$ per 100,000 , which is lower than the prevalence of $1-2$ per 100,000 shown for all SMA. ${ }^{5}$ Infants with
Correspondence: Andrew J Lloyd Acaster Lloyd Consulting Ltd., I6 Upper Woburn Place, London WCIHOBS, UK Tel +44203978 I68I

Email andrew.lloyd@acasterlloyd.com 
SMA Type I typically have onset of clinical signs by 6 months of age, never develop the ability to sit independently and generally do not survive beyond the first 2 years if no intervention is provided. ${ }^{3}$ SMA Type II is characterized by onset between 7 and 18 months of age. Patients develop the ability to sit independently and some are able to stand with assistance, but they do not develop the ability to walk independently. SMA Type III is typically diagnosed after 18 months of age, patients develop the ability to walk unaided but some lose the ability to walk at some point. Onset of SMA Type IV is typically in the second or third decade of life; motor impairment is mild without respiratory problems. ${ }^{6}$

Nusinersen was the first treatment targeting the underlying cause of disease approved by the FDA, EMA and other countries for patients with SMA. Nusinersen has demonstrated significant and clinically meaningful efficacy on the achievement of motor milestones and measures of motor function across a broad spectrum of SMA, and on survival endpoints in infantile-onset SMA. ${ }^{7-12}$

The efficient use of health care resources is a key consideration of decision-makers around the world, and so they commonly rely upon cost-effectiveness analysis to guide such decisions. In cost-effectiveness analysis, the benefits of treatments can be considered in terms of their impact on length of life and quality of life, estimated in terms of quality-adjusted life years (QALYs). ${ }^{13}$ Quality of life data for the estimation of QALYs reflects the value or utility of a health state on a scale from 0 (dead) to 1 (full health). A negative utility value represents a health state that is considered to be worse than dead. The UK weights for the EQ-5D for example range between 1 and $-0.594 .{ }^{14}$ These values are typically derived from the general public because it is felt that their priorities should be reflected by decision-makers. Standardised tools exist for the estimation of utilities - such as the EQ-5D. ${ }^{15}$ The QALY approach is widely used as a generic basis for measuring improvements in health and to support cost-effectiveness analyses. However, the EQ-5D is often criticised for a lack of sensitivity and measurement validity in certain disease areas. ${ }^{16}$ The existence of states worse than dead is also criticised by some people, although arguably these criticisms are not based on evidence.

Our searches identified one article reporting utility values for SMA $;{ }^{17}$ this study conducted in Spain reports a utility value of -0.012 for Type II SMA and a value of 0.158 for all types of SMA overall, estimated from caregiver EQ5D-3L proxy-reports. However, no other published utility values for different severities of Type I or II SMA were identified when this work was initiated. In rare diseases such as SMA, data regarding health-related quality of life (HRQoL) can be very hard to capture, especially if the information is not routinely measured in clinical trials. This is partly because of the rarity of the condition, but also due to the age profile of patients, and the fact that existing generic measures of utility have not been validated for use in infants and do not have UK value sets for children. In addition, even if HRQoL data are collected in clinical trials, in rare diseases, it is common for insufficient data to have been captured to allow estimation of utilities for all health states in an economic model. The NICE reference case ${ }^{18}$ states a preference for utilities for adults to be collected directly from patients using the EQ-5D; however, no specific instrument recommendation is given for collecting utilities for infants and pediatrics. For these reasons it is widely recognised that there are often gaps in the evidence base for assessing cost-effectiveness of treatments for rare diseases, ${ }^{19}$ particularly in pediatric conditions and so additional work is commonly needed which may rest upon expert opinion. Some recent cost-effectiveness analyses of treatments for pediatric conditions have included utilities partly derived from expert opinion for the reasons described above. ${ }^{20,21}$ In the current study, additional data on HRQoL in patients with different severities and types of SMA were needed in economic evaluations and in order to assess the cost-effectiveness of new therapies in SMA.

The present study was designed to address some of the gaps in the evidence in terms of HRQoL data to be used in an economic model of treatment for SMA. The values derived in the current study were reported in a recent cost-effectiveness evaluation of nusinersen in Sweden; ${ }^{22}$ however, the utility methods used were not fully described. This paper aims to describe the full methodology of the utility collection.

\section{Materials And Methods}

The study was designed to capture health utilities associated with treatment in SMA through the judgement of expert physicians who participated in SMA research efforts. This study was designed as an initial exploration of utilities so that values could be captured for all states in an economic model. Case histories were developed matching the definition of states in models designed to measure the cost-effectiveness of nusinersen for the treatment of SMA Types I and II. It was not possible to collect utility 
data for all model states from SMA patients or their families partly because of the rarity of the condition. The cost-effectiveness model included ten health states for Type I disease and seven states for Type II disease. Realistically, families could only be asked to judge the severity of the states that their child has experienced, which would only be a subset of all possible states. Therefore, for consistency, this initial study used the views of physicians only. The clinical experts were asked to review each case history and provide a proxy judgement of its impact on HRQoL using standardized measures. The study methods are summarized in Figure 1. The study was conducted in accord with the ethical standards of the Helsinki Declaration of 1975. As no data were collected from patients or caregivers, the study did not require review and approval by an independent review board or ethics committee.

\section{Development Of Case Histories}

The case histories describing different severities of SMA Types I and II were developed from a number of sources of information. A review of the literature was undertaken in order to explore how HRQoL is affected in SMA. Literature was identified that described the clinical manifestation of the disease and the range of symptoms and loss of functioning that patients can experience. The literature review identified very few studies detailing the HRQoL impact of SMA, in particular, a dearth of HRQoL information was found for Type I SMA. ${ }^{8,23-30}$ Studies were reviewed and any information pertaining to symptoms, functioning or HRQoL was extracted and summarized in a table for each type of SMA. The literature review was also used to develop an interview guide to be used in interviews with clinical experts.

\section{Expert Interviews}

Individual interviews were conducted with five clinical experts in SMA who participated in SMA research efforts in the UK. The experts were asked to describe a typical infant or child with different types of SMA in terms of the symptoms and functional limitations they might experience and to describe the HRQoL of infants or children with different types of SMA. In addition, experts were asked about the typical course of the disease for each type of SMA. Experts were also asked about the treatment options they currently have available and about three specific interventions in more detail: ventilation, scoliosis surgery and nasogastric/gastric tube.

\section{Case study development}

Literature review

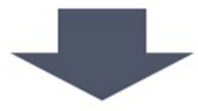

Interviews with clinical experts

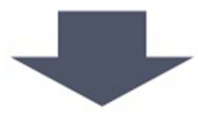

Draft case studies

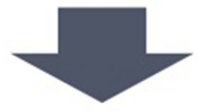

Expert review of case studies

\section{Valuation of case studies}

Interviews with clinical experts: Valuation of each case study using

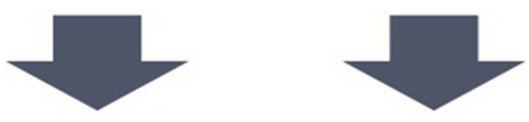

$E Q-5 D$ PedsQL NMM (baseline only)

Figure I Study methods flow chart.

\section{Draft Case Studies}

The interview results and literature review were used to draft case studies describing a child in each of the health states in the economic models for nusinersen for treatment of Type I and II SMA. The Type I health states were: no improvement (baseline), worsened after treatment, improvement following treatment, sits without support (reclassified as Type II), stands with assistance, walks 
with assistance, stands/walks unaided (reclassified as Type IIIa), ventilation, gastric/nasogastric tubes placement and scoliosis surgery. The Type II states were no improvement (baseline), worsened after treatment, mild increase in Hammersmith Functional Motor Scale-Expanded (HFMSE) score following treatment, moderate increase in HFMSE score following treatment, stands/walks with assistance, stands/walks unaided (reclassified as Type IIIa), loss of ambulation with/without assistance. The draft case studies were reviewed by clinical experts in SMA. Only minor changes to the states were made on the basis of this feedback. A statement was added to the improvement states about the HFMSE score improving. An example case study is shown in Figure 2 for the Type I baseline health state; each health state was described in a case study following the same format as shown in Figure 2.

\section{Valuation}

Five clinical experts in SMA in the UK participated in interviews to evaluate the case studies, including two who took part in the initial case study development interviews. Experts were sent the case studies prior to the interview; all interviews were conducted by telephone. Experts were asked to read each case study and to draw on their clinical experience to try to imagine how patients would be affected by that severity of SMA.

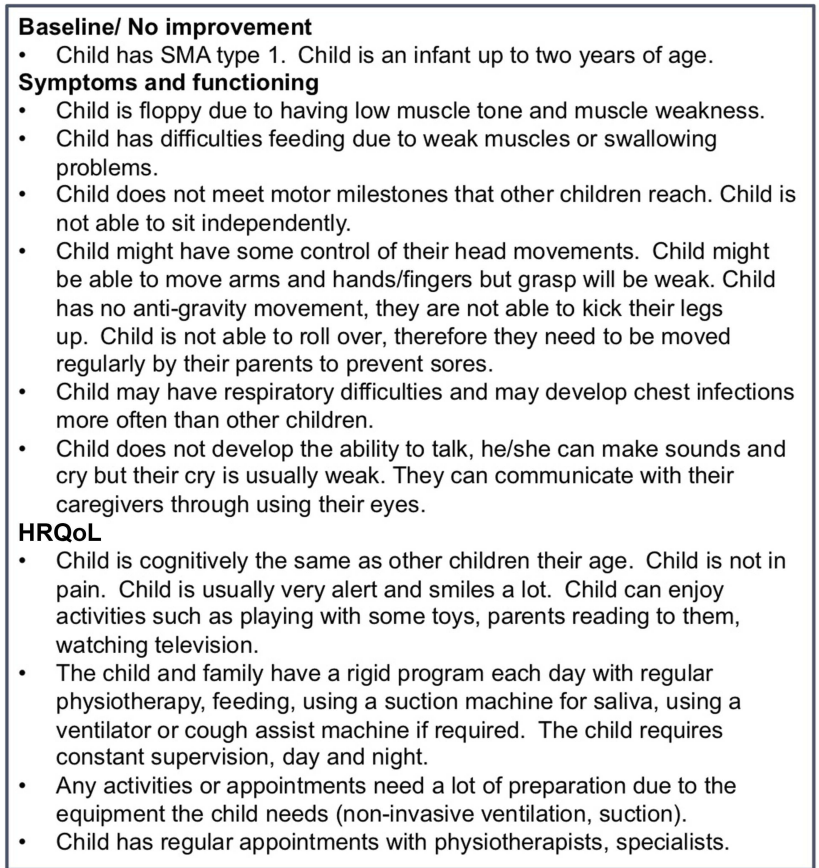

Figure 2 Example case study description (SMA Type I baseline).
After having read each case study, they were then asked to provide a proxy assessment to reflect how the quality of life of the child described in the case study would be affected. To provide the proxy assessment of HRQoL, they completed the EQ-5D- $\mathrm{Y}^{31}$ for all case studies and the Pediatric Quality of Life Inventory (PedsQL) NeuroMuscular Module (NMM) scale ${ }^{32}$ for the two baseline states (Type I and II "no improvement" states). The EQ-5D-Y is a child-version of the EQ-5D-3L and is a generic instrument that assesses five aspects of the HRQoL of a child including mobility (walking about); looking after myself (problems washing or dressing); usual activities (school, hobbies, sports, etc.); pain or discomfort and feeling worried sad or unhappy. Each dimension in the questionnaire is assessed as either "no problems", "some problems" or "a lot of problems". The EQ-5D-Y scored using UK weights ranges from 1 to $-0.594 .{ }^{14}$ The PedsQL NMM is a specific version of the PedsQL (a pediatric quality of life measure) designed to assess the impact of neuromuscular conditions including SMA. The questionnaire consists of three domains each scored on a 0-100 scale: "About my Neuromuscular Disease", a 17-item subscale assessing symptoms and functioning, "Communication", three items assessing the patient's ability to communicate with others about their condition, and "About our Family Resources", five items relating to the family's activities and resources. Each item is rated from "never" to "almost always".

\section{Analysis}

One clinician had some difficulties providing a rating for some domains of the EQ-5D-Y for some case studies. This was particularly related to the more subjective domains of pain/discomfort and emotional functioning. The clinician felt it was difficult to know how the patient feels, partly due to their young age and lack of ability to communicate. For these health states, the clinician provided two answers to some domains (eg, they would either have no pain/ discomfort or minimal pain/discomfort). In order to reflect this uncertainty, for these health states two index scores were calculated for this clinician, one using the less severe response and one using the more severe response; both index scores were included in the calculation of the mean score and standard deviation for those health states.

The data were scored using standard methods for each instrument. Responses on the EQ-5D are converted into a single index value. The EQ-5D-Y data were scored using the adult EQ-5D-3L tariff because there is no tariff 
currently available for the EQ-5D-Y. The data for each case study were summarized using descriptive statistics (mean and standard deviation) for each instrument.

\section{Results}

The experts who took part in the valuation interviews had a mean of 14 years' experience working with SMA patients and saw an average of 61 patients with SMA per year. Experts included two consultant paediatric neurologists, a clinical academic physician, a specialty doctor in paediatric neurology and a research physiotherapist. At the time of the study, all experts were all based in the UK.

Table 1 shows the mean utility values for the SMA Type I health state descriptions assessed by EQ-5D-Y and quality of life scores from the PedsQoL NMM (baseline state only). The table shows quite substantial differences in scores between some of the states. It is particularly noteworthy that most of the Type I states have a utility score below zero indicating a state considered to be worse than dead. The scores for the states range from -0.24 (Type I patient that has worsened from baseline) to 0.71 (a Type I patient that has shown such a substantial improvement in functioning that they have been reclassified as a Type IIIa patient).

Table 2 shows the mean utility values for the SMA Type II health state descriptions assessed by EQ-5D-Y and quality of life scores from the PedsQoL NMM (baseline state only). The results show differences in utility values between each health state, with baseline (0.04) and mild improvement (0.04) valued equally, and an increase of 0.06 moving from mild to moderate improvement $(0.10)$. There is a large increase in utility values for the standing/ walking states $(0.39$ and 0.72$)$. The loss of ambulation state is valued as worse than baseline $(-0.12)$.

It is also worth noting that the standard deviations around the mean scores are quite low, despite the fact that the study included only a small number of experts. This suggests a reasonable degree of agreement between the experts in their rating of the states.

\section{Discussion}

This study is the first to report utilities for health states describing different severities of SMA Types I and II in infantile-onset and later-onset (pediatric) patient populations, respectively. The results indicate the significant burden experienced by infants and pediatrics with SMA. The utility values elicited in this study have been used in a cost-effectiveness evaluation of nusinersen and provide an alternative to utilities estimated by mapping PedsQL data. ${ }^{22}$ The utility values from this study were used as important base-case model input parameters in the cost-effectiveness evaluation for nusinersen in both infantile-onset and later-onset SMA.

In order to capture utilities in this rare disease, methods were developed to collect data based on expert opinion rather than directly from patients. Detailed case study descriptions were developed for each health state. The

Table I Type I Health States And Associated HRQoL Scores

\begin{tabular}{|c|c|c|c|}
\hline \multirow[t]{3}{*}{ Health State } & \multicolumn{3}{|l|}{$N=5$} \\
\hline & \multirow{2}{*}{$\frac{\text { EQ-5D }}{\text { Mean (SD) }}$} & \multicolumn{2}{|l|}{ PedsQoL NMM } \\
\hline & & Domain & Mean (SD) \\
\hline \multirow[t]{3}{*}{ Baseline } & \multirow[t]{3}{*}{$-0.12(0.19)$} & About & $29.6(6.5)$ \\
\hline & & Communication & $9.4(8.8)$ \\
\hline & & Family & $24.1(13.9)$ \\
\hline Worsened & $-0.24(0.14)$ & & \\
\hline Improvement & $-0.17(0.17)$ & & \\
\hline Sits without support ${ }^{\mathrm{a}}$ & $-0.04(0.12)$ & & \\
\hline Stands with assistance & $0.04(0.09)$ & & \\
\hline Walks with assistance ${ }^{\mathrm{b}}$ & $0.52(0.22)$ & & \\
\hline Stands/walks unaided ${ }^{b, c}$ & $0.71(0.14)$ & & \\
\hline SMA after scoliosis surgery & $-0.22(0.22)$ & & \\
\hline Gastric/NG tube & $-0.17(0.17)$ & & \\
\hline Requires ventilation & $-0.33(0.27)$ & & \\
\hline
\end{tabular}

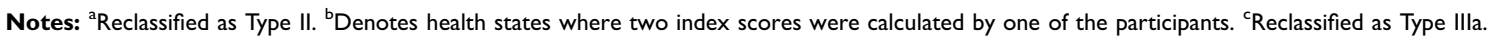


Table 2 Type II Health States And Associated HRQoL Scores

\begin{tabular}{|c|c|c|c|}
\hline \multirow[t]{3}{*}{ Health State } & \multicolumn{3}{|l|}{$N=5$} \\
\hline & \multirow{2}{*}{$\begin{array}{l}\text { EQ-5D } \\
\text { Mean (SD) }\end{array}$} & \multicolumn{2}{|l|}{ PedsQoL NMM } \\
\hline & & Domain & Mean (SD) \\
\hline \multirow[t]{3}{*}{ Baseline } & \multirow[t]{3}{*}{$0.04(0.10)$} & About & $43.8(7.3)$ \\
\hline & & Communication & $56.7(16.0)$ \\
\hline & & Family & $44.0(8.0)$ \\
\hline Worsened & $-0.13(0.06)$ & & \\
\hline Mild improvement & $0.04(0.11)$ & & \\
\hline Moderate improvement & $0.10(0.09)$ & & \\
\hline Stands/walks with assistance* & $0.39(0.29)$ & & \\
\hline Stands/walks unaided* & $0.72(0.12)$ & & \\
\hline Loss of ambulation with/without assistance* & $-0.12(0.16)$ & & \\
\hline
\end{tabular}

Note: *Denotes health states where two index scores were calculated for one of the participants.

resulting HRQoL data had a logical structure to it, in that the scores were higher for improved health states (apart from the "improvement" state for Type I SMA). These values provide an indication of the HRQoL burden and the potential gains in HRQoL associated with improvements in the disease and the patients' level of function.

The methods used in the present study allowed us to estimate utilities for each health state, but there are some important limitations to highlight. This was not a prospective observational study in families affected with SMA which would be the gold standard approach. The accuracy of the HRQoL results was dependent on the validity or accuracy of the case study descriptions, and the ability of the experts to provide an accurate proxy assessment of HRQoL. This study did not capture patients' or parents' perception of HRQoL, but rather the burden was judged by health care professionals. Their views regarding the impacts of different aspects of SMA, such as the need for tracheostomy, may vary in important ways from parents and the children themselves and this is the most significant limitation of this work. Also, there is some previous research which suggests that pediatricians may underestimate the level of pain and discomfort that children experience compared to the children's parents. The level of agreement between pediatricians and parents is found to be lower on subjective domains of HRQoL compared to more objective measures. ${ }^{33,34}$ The use of the case study vignettes may help to provide more consistency and reduce this potential for bias. Physicians are also able to draw on their experience of treating numerous patients with SMA, as opposed to a parent's experience of caring for just their child. This may allow us to avoid some bias that parental judgement of HRQoL may introduce. Secondly, the manifestation of SMA is heterogeneous but the descriptions of SMA states are by necessity a simplification and do not reflect this variability. Thirdly, it was only possible to recruit a small group of expert physicians; however, while the group was small, their ratings were quite consistent. Lastly, the assessment of HRQoL used the EQ$5 \mathrm{D}-\mathrm{Y}$ which has not been proven as valid in children as young as some patients with SMA (eg, infantile-onset patient population). However, no other alternatives currently exist for the assessment of HRQoL in such young children or infants. Despite these limitations, the study has produced data with face validity. The authors believe that this study is a starting point for understanding HRQoL in SMA which could usefully be followed with further prospective, observational or clinical trial research. Future work can improve on the limitations in this research and include potential caregiver and patient interviews.

\section{Conclusions}

In this study, clinician expert opinion has been used to describe SMA case studies which have then been rated in terms of their impact on HRQoL. The study produced results with face validity; however, the limitations of these methods have also been discussed. Prospective research is needed to better understand the HRQoL burden of SMA for children and their caregivers.

\section{Acknowledgments}

The abstract of this paper was presented at the 2017 International Society for Pharmacoeconomics and Outcomes 
Research (ISPOR) conference as a poster presentation. The poster's abstract was published in "Poster Abstracts" in Value in Health (2017) 20(9): A559 https://www.valueinhealthjour nal.com/article/S1098-3015(17)31245-7/fulltext.

\section{Author Contributions}

All authors contributed to data analysis, drafting and revising the article, gave final approval of the version to be published and agree to be accountable for all aspects of the work.

\section{Funding}

Biogen sponsored this research project which was conducted by Acaster Lloyd Consulting Ltd, an independent research consultancy. Biogen were involved in the study design and development of the manuscript.

\section{Disclosure}

RT is an employee of Biogen. MT was an employee of Biogen at the time when this study was conducted. AJL and $\mathrm{KG}$ are employees of Acaster Lloyd Consulting Ltd. The authors report no other conflicts of interest in this work.

\section{References}

1. Lally C, Jones C, Farwell W, Reyna SP, Cook SF, Flanders WD Indirect estimation of the prevalence of spinal muscular atrophy Type I, II, and III in the United States. Orphanet $J$ Rare Dis. 2017;12(1):175. doi:10.1186/s13023-017-0724-Z

2. Zerres K, Rudnik-Schöneborn S. Natural history in proximal spinal muscular atrophy: clinical analysis of 445 patients and suggestions for a modification of existing classifications. Arch Neurol. 1995;52 (5):518-523. doi:10.1001/archneur.1995.00540290108025

3. D'Amico A, Mercuri E, Tiziano FD, Bertini E. Spinal muscular atrophy. Orphanet J Rare Dis. 2011;6(1):71. doi:10.1186/1750-1172-6-48

4. Ogino S, Leonard DG, Rennert H, Ewens WJ, Wilson RB. Genetic risk assessment in carrier testing for spinal muscular atrophy. Am J Med Genet A. 2002;110(4):301-307. doi:10.1002/ajmg.10425

5. Verhaart IE, Robertson A, Wilson IJ, et al. Prevalence, incidence and carrier frequency of 5q-linked spinal muscular atrophy - a literature review. Orphanet J Rare Dis. 2017;12(1):124. doi:10.1186/s13023-017-0671-8

6. Wang $\mathrm{CH}$, Finkel RS, Bertini ES, et al. Consensus statement for standard of care in spinal muscular atrophy. J Child Neurol. 2007;22 (8):1027-1049. doi:10.1177/0883073807305788

7. Finkel RS, Mercuri E, Darras BT, et al. Nusinersen versus sham control in infantile-onset spinal muscular atrophy. New Engl J Med. 2017;377(18):1723-1732. doi:10.1056/NEJMoa1702752

8. Finkel RS, Chiriboga CA, Vajsar J, et al. Treatment of infantile-onset spinal muscular atrophy with nusinersen: a phase 2, open-label, doseescalation study. Lancet. 2016;388(10063):3017-3026. doi:10.1016/ S0140-6736(16)31408-8

9. Mercuri E, Finkel R, Kirschner J, et al. Efficacy and safety of nusinersen in children with later-onset spinal muscular atrophy (SMA): end of study results from the phase 3 CHERISH study. Neuromuscul Disord. 2017;27: S210.
10. Mercuri E, Darras BT, Chiriboga CA, et al. Nusinersen versus sham control in later-onset spinal muscular atrophy. $N$ Engl $J$ Med. 2018;378(7):625-635. doi:10.1056/NEJMoa1710504

11. Darryl C, Hwu WL, Reyna SP, et al. Interim efficacy and safety results from the phase 2 NURTURE study evaluating nusinersen in presymptomatic infants with spinal muscular atrophy (S46. 003). Neurology. 2017;88(16 Supplement):S46-003.

12. Pane M, Palermo C, Messina S, et al. Nusinersen in type 1 SMA infants, children and young adults: preliminary results on motor function. Neuromuscul Disord. 2018;28(7):582-585. doi:10.1016/j. nmd.2018.05.010

13. Drummond MF, Sculpher MJ, Claxton K, Stoddart GL, Torrance GW. Methods for the Economic Evaluation of Health Care Programmes. Oxford university press; 2015.

14. Dolan P. Modeling valuations for EuroQol health states. Med Care. 1997;35(11):1095-1108. doi:10.1097/00005650-199711000-00002

15. Herdman M, Gudex C, Lloyd A, et al. Development and preliminary testing of the new five-level version of EQ-5D (EQ-5D-5L). Qual Life Res. 2011;20(10):1727-1736. doi:10.1007/s11136-011-9903-x

16. Brazier J, Ara R, Rowen D, Chevrou-Severac H. A review of generic preference-based measures for use in cost-effectiveness models. PharmacoEconomics. 2017;35(1):21-31. doi:10.1007/s40273-017-05 $45-\mathrm{x}$

17. López-Bastida J, Peña-Longobardo LM, Aranda-Reneo I, Tizzano E, Sefton M, Oliva-Moreno J. Social/economic costs and health-related quality of life in patients with spinal muscular atrophy (SMA) in Spain. Orphanet J Rare Dis. 2017;12(1):141. doi:10.1186/s13023017-0695-0

18. National Institute for Health and Care Excellence (NICE). Guide to the Methods of Technology Appraisal. National Institute for Health and Care Excellence; 2013.

19. Gosling JP. Methods for Eliciting Expert Opinion to Inform Health Technology Assessment. Vignette Commissioned by the MRC Methodology Advisory Group. Medical Research Council (MRC) and National Institure for Health Research (NIHR); 2014.

20. National Institute for Health and Care Excellence (NICE). Burosumab for Treating X-Linked Hypophosphataemia in Children and Young People. National Institute for Health and Care Excellence; 2018.

21. National Institute for Health and Care Excellence (NICE). Asfotase Alfa for Treating Paediatric-Onset Hypophosphatasia. National Institute for Health and Care Excellence; 2017.

22. Zuluaga-Sanchez S, Teynor M, Knight C, et al. Cost effectiveness of nusinersen in the treatment of patients with infantile-onset and lateronset spinal muscular atrophy in Sweden. Pharmaco Economics. 2019;(2019:1-21.

23. O'Hagen JM, Glanzman AM, McDermott MP, et al. An expanded version of the Hammersmith Functional Motor Scale for SMA II and III patients. Neuromuscul Disord. 2007;17(9-10):693-697. doi:10.1016/j.nmd.2007.05.009

24. Klug C, Schreiber-Katz O, Thiele S, et al. Disease burden of spinal muscular atrophy in Germany. Orphanet J Rare Dis. 2016;11(1):58. doi:10.1186/s13023-016-0424-0

25. Bach JR, Vega J, Majors J, Friedman A. Spinal muscular atrophy type 1 quality of life. Am J Phys Med Rehabil. 2003;82(2):137-142. doi:10.1097/00002060-200302000-00009

26. De Sanctis R, Coratti G, Pasternak A, et al. Developmental milestones in type I spinal muscular atrophy. Neuromuscul Disord. 2016;26(11):754-759. doi:10.1016/j.nmd.2016.10.002

27. Kaufmann P, McDermott MP, Darras BT, et al. Observational study of spinal muscular atrophy type 2 and 3: functional outcomes over 1 year. Arch Neurol. 2011;68(6):779-786. doi:10.1001/archneurol.2010.373

28. Finkel RS, McDermott MP, Kaufmann P, et al. Observational study of spinal muscular atrophy type I and implications for clinical trials. Neurology. 2014;83:10-212. 
29. Qian Y, McGraw S, Henne J, Jarecki J, Hobby K, Yeh WS. Understanding the experiences and needs of individuals with spinal muscular atrophy and their parents: a qualitative study. BMC Neurol. 2015;15(1):217. doi:10.1186/s12883-015-0473-3

30. Montes J, Gordon AM, Pandya S, De Vivo DC, Kaufmann P. Clinical outcome measures in spinal muscular atrophy. $J$ Child Neurol. 2009;24(8):968-978. doi:10.1177/0883073809332702

31. Wille N, Badia X, Bonsel G, et al. Development of the EQ-5D-Y: a child-friendly version of the EQ-5D. Qual Life Res. 2010;19(6):875886. doi:10.1007/s11136-010-9648-y

32. Iannaccone ST, Hynan LS, Morton A, Buchanan R, Limbers CA, Varni JW. The PedsQL TM in pediatric patients with spinal muscular atrophy: feasibility, reliability, and validity of the pediatric quality of life inventory $^{\mathrm{TM}}$ generic core scales and neuromuscular module. Neuromuscul Disord. 2009;19(12):805-812. doi:10.1016/j.nmd.2009.09.009
33. Janse AJ, Sinnema G, Uiterwaal CS, Kimpen JL, Gemke RJ. Quality of life in chronic illness: perceptions of parents and paediatricians. Arch Dis Child. 2005;90(5):486-491. doi:10.1136/adc.2004.051722

34. Janse AJ, Gemke RJ, Uiterwaal CS, Van Der Tweel I, Kimpen JL, Sinnema G. Quality of life: patients and doctors don't always agree: a meta-analysis. J Clin Epidemiol. 2004;57(7):653-661. doi:10.1016/j. jclinepi.2003.11.013
ClinicoEconomics and Outcomes Research

\section{Publish your work in this journal}

ClinicoEconomics and Outcomes Research is an international, peerreviewed open-access journal focusing on Health Technology Assessment, Pharmacoeconomics and Outcomes Research in the areas of diagnosis, medical devices, and clinical, surgical and pharmacological intervention. The economic impact of health policy and health systems

\section{Dovepress}

organization also constitute important areas of coverage. The manuscript management system is completely online and includes a very quick and fair peer-review system, which is all easy to use. Visit http://www.dovepress.com/testimonials.php to read real quotes from published authors. 